\title{
Effect of sustained postnatal systemic inflammation on hippocampal volume and function in mice
}

\author{
Shadi N. Malaeb', Jonathan M. Davis', Ilka M. Pinz², Jennifer L. Newman³, Olaf Dammann ${ }^{4,5}$ and Maribel Rios ${ }^{3}$
}

\begin{abstract}
BACKGROUND: Premature infants are at risk for persistent neurodevelopmental impairment. Children born preterm often exhibit reduced hippocampal volumes that correlate with deficits in working memory. Perinatal inflammation is associated with preterm birth and brain abnormalities. Here we examine the effects of postnatal systemic inflammation on the developing hippocampus in mice.
\end{abstract}

METHODS: Pups received daily intraperitoneal injections of lipopolysaccharide (LPS) or saline between days 3 and 13 . Ex vivo magnetic resonance imaging (MRI) and microscopic analysis of brain tissue was performed on day 14. Behavioral testing was conducted at 8-9wk of age.

RESULTS: MR and microscopic analysis revealed a 15-20\% reduction in hippocampal volume in LPS-treated mice compared with controls. Behavioral testing revealed deficits in hippocampal-related tasks in LPS-treated animals. Adult mice exposed to LPS during the postnatal period were unable to select a novel environment when re-placed within a 1-min delay, were less able to remember a familiar object after a 1-h delay, and had impaired retention of associative fear learning after $24 \mathrm{~h}$.

CONCLUSION: Systemic inflammation sustained during the postnatal period contributes to reduced hippocampal volume and deficits in hippocampus-dependent working memory. These findings support the novel and emerging concept that sustained systemic inflammation contributes to neurodevelopmental impairment among preterm infants.

$\mathbf{P}$ reterm birth is a significant burden in the United States and worldwide (1). Over 56,000 preterm infants were born with very low birth weight in the United States in 2012 (2). Advances in perinatal and neonatal care have led to increased survival of preterm infants. However, many children born very preterm still suffer major neurodevelopmental impairment (NDI) that persists well into adulthood and manifests as poor executive function, suboptimal academic performance, attention deficits, and behavioral problems (3-5). A poor working memory contributes significantly to these deficits (6).
The hippocampus is a dynamic segment of the limbic system that is crucial for developing working memory during infancy $(7,8)$. Preterm infants who exhibited working memory deficits at 2 y corrected age showed smaller hippocampal volumes when measured by magnetic resonance imaging (MRI) of the brain at term equivalent age compared with preterm infants who developed without NDI (9). This is consistent with previous findings that the hippocampus is vulnerable to many insults affecting preterm infants (10).

Perinatal infection and inflammation play a role in the etiology of preterm birth and brain injury among preterm newborns (11). The systemic fetal inflammatory response can continue postnatally and further contribute to brain damage $(12,13)$. Intraperitoneal administration of lipopolysaccharide (LPS) has been used to induce sustained postnatal systemic inflammation in newborn mice (14). We wanted to investigate the effect of sustained postnatal systemic inflammation on the developing hippocampus and test the hypothesis that daily intraperitoneal administration of LPS is associated with reduced hippocampal volume at postnatal day 14 and with deficits in hippocampus-dependent working memory that persist into adulthood in mice.

\section{RESULTS}

\section{Orthometric Measures}

The wet brain weight of LPS-treated mice was reduced by $15 \%$ (LPS: $0.312 \pm 0.006 \mathrm{~g}, n=13$; PBS: $0.365 \pm 0.007 \mathrm{~g}, n=15 ; \mathrm{M} \pm$ SEM; $P<0.01$ ), and body weight by $22 \%$ (LPS: $5.14 \pm 0.26 \mathrm{~g}, n=$ 13; PBS: $6.60 \pm 0.26 \mathrm{~g}, n=15 ; \mathrm{M} \pm \mathrm{SEM}$; $P<0.01)$ compared with controls on postnatal day 14 . The brain to body weight ratios were not significantly different between groups. There were no significant differences in brain weights (PBS: $0.365 \pm 0.007 \mathrm{~g}$, $n$ $=15$; naïve: $0.381 \pm 0.004 \mathrm{~g}, n=9 ; \mathrm{M} \pm \mathrm{SEM} ; P=0.12$ ) or body weights (PBS: $6.599 \pm 0.258 \mathrm{~g}, n=15$; naïve: $6.756 \pm 0.184 \mathrm{~g}, n=$ 9; $\mathrm{M} \pm$ SEM; $P>0.6$ ) between sham control and naïve animals. Of note, mice injected with LPS often exhibited a shiny and full abdomen suggestive of ascites. Mice that died following LPS exposure often appeared wasted. However, there were no differences in the average body weights between survivors in the

\footnotetext{
'Department of Pediatrics, The Floating Hospital for Children at Tufts Medical Center, Boston, Massachusetts; ${ }^{2}$ Center for Molecular Medicine, Maine Medical Center Research Institute, Scarborough, Maine; ${ }^{3}$ Department of Neuroscience, Tufts University School of Medicine, Boston, Massachusetts; ${ }^{4}$ Department of Public Health \& Community Medicine, Tufts University School of Medicine, Boston, Massachusetts; ${ }^{5}$ Department of Obstetrics and Gynecology Perinatal Neuroepidemiology Unit, Hannover Medical School, Hannover, Germany. Correspondence: Shadi N. Malaeb (Shadi.Malaeb@DrexelMed.edu) 
LPS and control groups at $8 \mathrm{wk}$ of life (LPS: $21.53 \pm 0.94 \mathrm{~g}, n=$ 9; PBS: $21.35 \pm 0.60, n=14 ; \mathrm{M} \pm \mathrm{SEM} ; P>0.8$ ).

\section{Hippocampal Measures on Postnatal Day 14}

The hippocampal volume measured by MRI was reduced by $20 \%$ in the LPS compared with the control group on postnatal day 14 (7.53 \pm 0.46 vs. $9.42 \pm 0.17 \mathrm{~mm}^{3}$; $\mathrm{M} \pm$ SEM; $P<0.01$; Figure 1a). Moreover, bilateral periventricular porencephalic cysts were grossly evident on MRI in one of the mice in the LPS compared with none in the control group. This corresponded to regions of white matter loss. Microscopic analysis of the hippocampal region on matching rostral and caudal tissue sections showed a comparable $25 \%$ reduction in the area of the hippocampal region between the LPS compared with the control group on postnatal day 14 (17.48 \pm 0.71 vs. $23.29 \pm 0.24 \mathrm{~mm}^{2} ; \mathrm{M} \pm \mathrm{SEM} ; \mathrm{P}<0.01$; Figure $\left.1 \mathrm{~b}\right)$.

\section{Behavioral Analysis}

A total of 23 mice survived to adulthood (14 sham control and 9 LPS-exposed mice) and were tested between 8 and $9 \mathrm{wk}$ of life with a battery of neurobehavioral tests. Two mice, one in the LPS group and one in the control group, were excluded as outliers from final analysis because each consistently performed $>3$ SDs below average for its group.
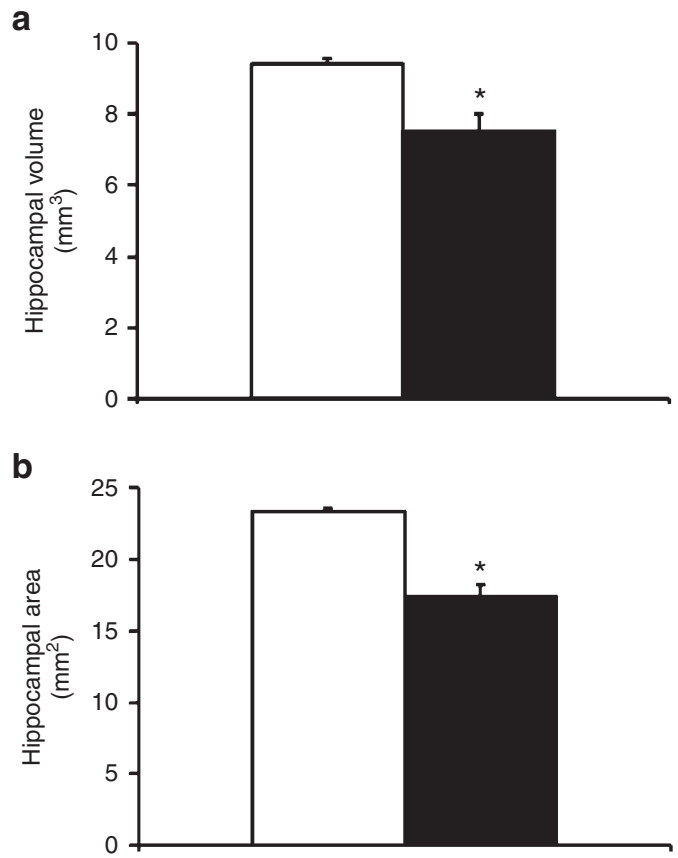

Figure 1. (a) Average volumes of the hippocampal formation calculated as the summation of both the right and left hippocampal regions in all of the sequential images from each brain multiplied by the section thickness $(27 \mu \mathrm{m})$. (b) Average areas of the hippocampal formation calculated by adding the areas of both the right and left hippocampal regions in rostral and caudal sections at matching levels from each brain. The hippocampal volume was reduced by $20 \%$ and its area was reduced by $25 \%$ in the lipopolysaccharide (LPS) group compared with the control group on postnatal day 14 ( $\mathrm{M} \pm \mathrm{SEM}$; ${ }^{*} P<0.01$ vs. control; control: open bars; LPS: solid bars).
Rota-Rod Performance in Adulthood

Mice in the control and LPS groups successfully tolerated comparable speeds of approximately $30 \mathrm{rpm}$ on the Rota-Rod $(31.2 \pm 1.6$ vs. $31.4 \pm 1.3 \mathrm{rpm} ; \mathrm{M} \pm \mathrm{SEM} ; P>0.9)$ and latencies to fall $(241.7 \pm 12.6$ vs. $245.6 \pm 13.1 \mathrm{~s} ; \mathrm{M} \pm \mathrm{SEM} ; P>0.9)$. These speeds were similar to speeds tolerated by juvenile naïve mice $(31.9 \pm 2.6 \mathrm{rpm} ; P>0.8)$.

\section{Cognitive Performance in Adulthood}

Spontaneous alternation on a T-maze. Mice in both the LPS and the control groups showed comparable activity reflected by the time lapsed to move out of the base chamber (PBS: $16.4 \pm 3.2$; LPS: $19.9 \pm 2.8 \mathrm{~s} ; \mathrm{M} \pm \mathrm{SEM}$; $P>0.4)$. Mice in the control group spontaneously alternated between the arms of the T-maze on $77.6 \pm 5.6 \%$ of the trials. This is consistent with the report on spontaneous alternation of healthy C57BL6 mice on the T-maze of approximately $80 \%$ (15). In contrast, mice in the LPS group alternated between the arms of the T-maze on $53.3 \pm 4.6 \%$ of the time $(P<0.01$; Figure $2 \mathrm{a})$, which is considered a random association.

Novel object recognition. Mice in both the control and the LPS groups demonstrated similar levels of locomotor activity with no significant differences in the number of midline chamber crossovers (e.g., number of crosses per minute; PBS: $6.6 \pm 0.7$; LPS: $8.5 \pm 0.8 ; \mathrm{M} \pm \mathrm{SEM} ; P=0.09)$ or in the total time spent exploring the objects $(32.4 \pm 12.9 \mathrm{~s}$ in the PBS group vs. $36.3 \pm 11.4 \mathrm{~s}$ in the LPS group; $\mathrm{M} \pm \mathrm{SEM}$; $>$ 0.6). However, mice in the LPS group were less able to discriminate a novel object compared with control mice, with discrimination factors of $0.72 \pm 0.02$ for the control group and $0.65 \pm 0.03$ for the LPS group ( $\mathrm{M} \pm \mathrm{SEM}$; $<0.05$; Figure $2 \mathrm{~b})$. Mice in the sham control group showed similar discriminatory ability as naïve mice.

Contextual fear conditioning test. Mice in both the control and the LPS groups showed comparable locomotor activity as defined by the number of midline chamber crossovers $(6.4 \pm 0.7$ bouts/min vs. $6.5 \pm 0.9)$. Mice in both groups froze significantly more following each consecutive shock compared with baseline, indicating successful acquisition of learning after presentation of the shock stimulus $(F=67.9, d f=4, P$ $<0.01$ from baseline; Figure 2c). When returned to the same chamber $24 \mathrm{~h}$ later, mice in both groups froze significantly more throughout the observation period compared with baseline, indicating memory of the fearful context $(P<0.01$; Figure 2d). Post hoc analysis indicated that control mice demonstrated higher levels of freezing throughout the observation period than the LPS group $(P<0.01$; Figure $2 \mathrm{~d})$. Lower levels of freezing indicated weaker retention of the associative learning acquired $24 \mathrm{~h}$ earlier. No differences were detected between male and female mice in any of the cognitive tests.

\section{DISCUSSION}

The results of our study indicate that repeated systemic exposure to LPS during the early postnatal period is associated with 
a

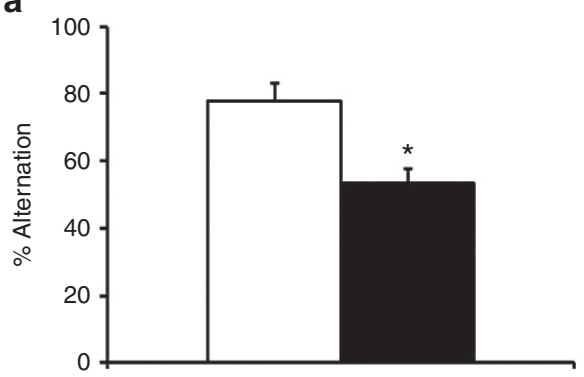

C

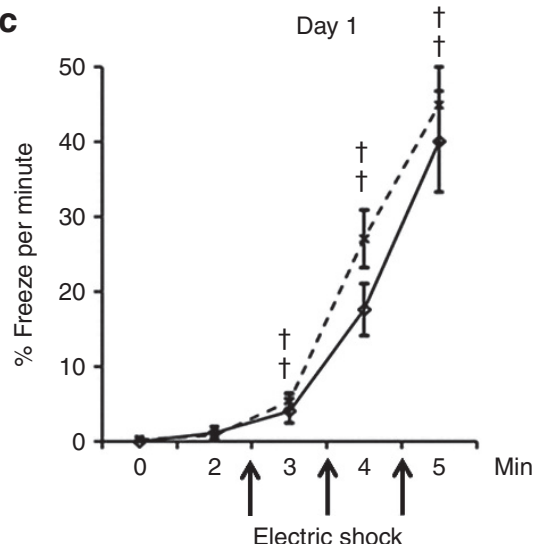

b

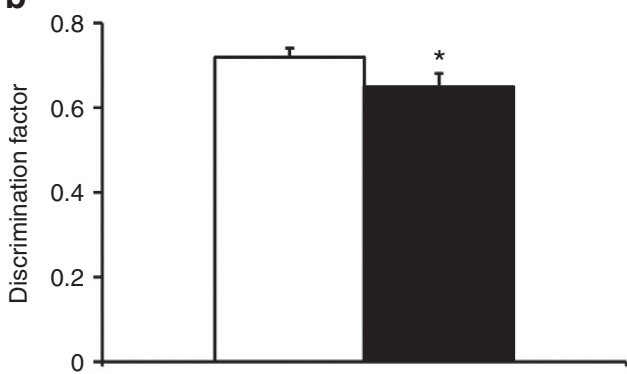

d

Day 2

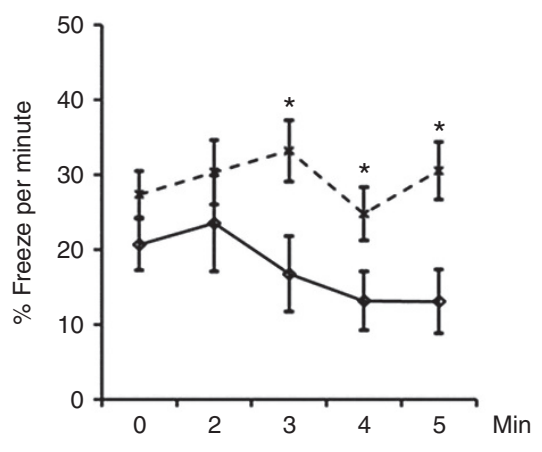

Figure 2. Cognitive assessment of mice between 8 and $9 w k$ of life with a battery of tests of hippocampus-dependent working memory tasks that include spontaneous alternation on a T-maze (a; control: open bars; lipopolysaccharide (LPS): solid bars), novel object recognition (b; control: open bars; LPS: solid bars), and contextual fear conditioning (c and d; control: dotted line; LPS: solid line). Mice that were injected daily with LPS during the postnatal period were not able as adults to register and select a novel environment when re-placed within a 1-min delay on a T-maze, were less able to remember a familiar object after a 1-h delay, and showed impaired retrieval and retention of associative learning after $24 \mathrm{~h}$ (d), despite adequate initial acquisition of associative learning to the fearful stimulus (c; $\mathrm{M} \pm \mathrm{SEM} ;{ }^{\dagger} P<0.01$ vs. 1 -min on day $1 ;{ }^{*} P<0.05$ vs. control).

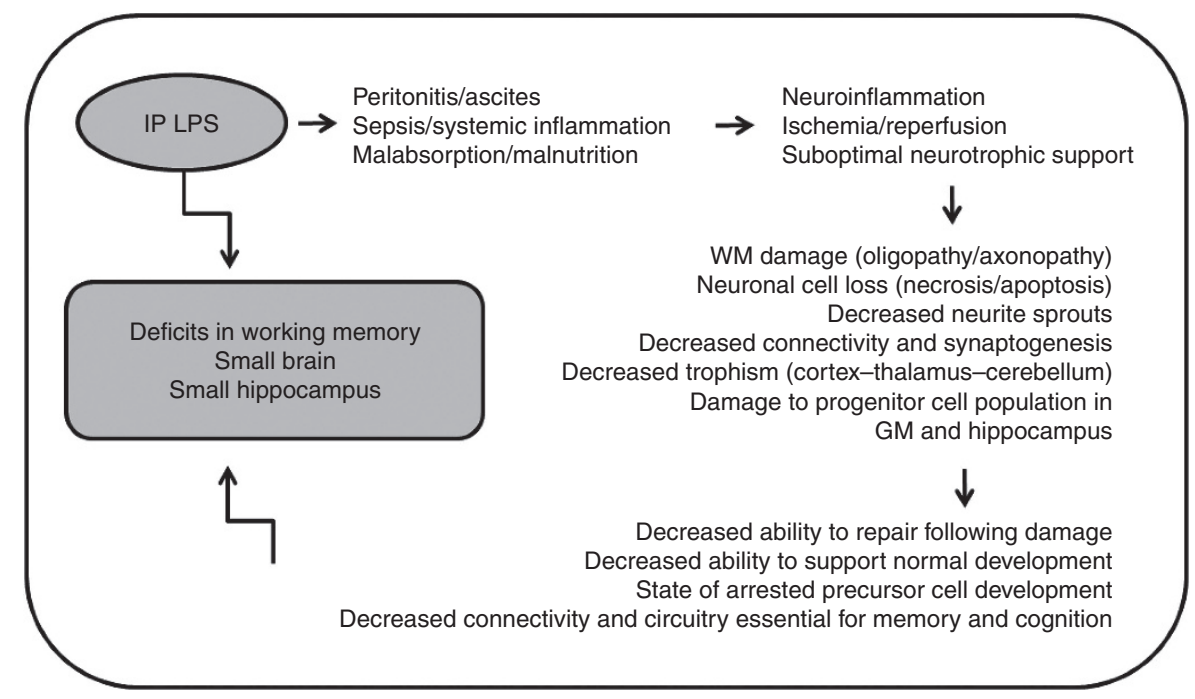

Figure 3. Proposed mechanisms that may have resulted in the observed association between postnatal exposure to lipopolysaccharide (LPS) and subsequent impairment of hippocampal development in mice. GM, germinal matrix; WM, white matter.

reduced brain weight and hippocampal volume in juvenile mice as well as concomitant deficits in hippocampus-dependent working memory in adult animals. Specifically, the volume of the hippocampal formation was reduced by $20 \%$ in LPS-exposed mice compared with controls when measured ex vivo using MRI and confirmed by histology. Furthermore, mice treated with LPS were not able to register and select a novel environment and exhibited deficient memory of a familiar object when tested as adults. Fear conditioning studies indicate that LPS treatment was associated with impaired retrieval and retention of hippocampus-dependent associative learning after $24 \mathrm{~h}$, despite adequate initial acquisition of associative learning 


\section{Articles | Malaebe tal.}

to the fearful stimulus. There were no appreciable differences in motor performance or baseline hyperactivity between the two groups that would have confounded subsequent tests.

The role of sustained postnatal systemic inflammation in the pathogenesis of perinatal brain injury is an emerging concept in developmental neurobiology (11-13). Wang and colleagues $(14,16)$ originally developed a model of sustained postnatal systemic inflammation by administering LPS $(0.3 \mathrm{mg} / \mathrm{kg})$ or a toll-like receptor (TLR) agonist intraperitoneally to C57BL/6 newborn mice daily from postnatal days 3 to 11 , followed by anatomical brain examination and functional analysis at postnatal days 12 and 50, respectively. They reported significant induction of a systemic inflammatory response in LPS-treated mice, with serum interleukin- 6 increasing by over 1,000 -fold. These investigators also found significant reductions in cerebral gray matter and subcortical white matter volume, decreased hippocampal neuronal density, and an increase in cerebral microglia density at postnatal day 12 . However, LPS-treated mice performed similarly to controls in the fear conditioning test on postnatal day 50 . Of note, there was no increase in mortality and brain weight was marginally reduced only after TLR agonist exposure and not after LPS exposure (16). These findings suggest that chronic subclinical inflammation hampers development of white and gray matter in early life (14). In contrast, our study indicates stronger and longer-lasting effects of the same dose of LPS (0.3 mg/kg i.p.; Escherichia coli 055:B5) with $50 \%$ mortality, $16 \%$ reduction in brain weight, and a significant impairment in hippocampus-dependent working memory in adult mice. Our findings suggest a greater severity of illness that may be attributed to a heightened potency of LPS secondary to variation between different lots of E. coli, and/or possibly to a more homogeneous distribution of the LPS after ultrasonication.

Favrais et al. (17) designed a model of sustained postnatal systemic inflammation involving intraperitoneal injections of IL-1 $\beta$ in newborn mice between postnatal days 1 and 5 . They reported a transient increase in the density of microglia in the neopallium, but no detectable effects on neocortical cell death or in neuronal and astrocytic cell density. A long-lasting myelination defect was characterized by increased numbers of nonmyelinated axons and a reduced diameter of myelinated axons. A decreased density of myelinating oligodendrocytes and an increased density of oligodendrocyte progenitors suggest a partial blockade in the oligodendrocyte maturation process. These abnormalities correlated with reduced white matter fractional anisotropy on diffusion tensor MRI. Cognitive testing at $1 \mathrm{mo}$ of age showed persistent working memory deficits in mice exposed to IL-1 $\beta$ in the neonatal period manifesting as impaired novel object and displaced object recognition. There were no appreciable differences in motor performance in an open field between the two groups. These findings indicate that systemic inflammation sustained in the neonatal period is associated with injury to the developing brain that lasts well into adulthood in mice. Our results are consistent with those of Favrais and associates, and further indicate that the hippocampus is primarily affected following postnatal systemic inflammation since mice exposed to sustained systemic inflammation during the early postnatal period exhibited deficits in retrieval of associative learning during contextual fear conditioning (CFR), a task that depends on intact hippocampal function.

Our findings add to previous findings that perinatal systemic inflammation causes persistent abnormality in the developing hippocampus resulting in subsequent learning disabilities. A single neonatal intraperitoneal injection of LPS $(0.1 \mathrm{mg} / \mathrm{kg})$ caused long-lasting changes in hippocampal and cortical $\mathrm{N}$-methyl-D-aspartate receptor mRNA expression, impaired learning and memory, and a trend towards inferior performance in the fear conditioning in adult animals (18). An intracerebral injection of a larger dose of LPS $(1 \mathrm{mg} / \mathrm{kg})$ given to newborn rats on postnatal day 5 led to dramatic learning deficits and reduced anxiety-like responses in the elevated plus-maze task, as well as a reduction in hippocampal volume, neuronal density, axonal integrity, and sustained inflammatory responses in the adult rat hippocampus following neonatal LPS exposure (19). LPS administered intraperitoneally to pregnant rats on embryonic days 15 and 16 was associated with a reduction in presynaptic input to the hippocampus and a compensatory enhancement in postsynaptic response and pyramidal cell excitability (20). Recently, Smith et al. (21) showed that intraperitoneal administration of LPS on day 5 to newborn mice dysregulates neurogenesis in the developing hippocampus and results in a mixed M1/M2 microglial response in the hippocampus with increased expression of both $\mathrm{M} 1$ and $\mathrm{M} 2$ genes on day 7, but only of M1 genes on day 21 .

Perinatal infection and inflammation play an important role in the etiology of premature birth and in the pathogenesis of preterm brain injury (11). The systemic inflammatory response can continue postnatally $(12,13)$. Elevated levels of inflammation-related proteins, especially when sustained over multiple days, were associated with developmental impairment at 2 y of age compared with elevations present for only $1 \mathrm{~d}(22,23)$. Brain MRI of preterm infants who exhibited working memory deficits at 2 y corrected age showed smaller hippocampal volume at term equivalent age compared with preterm infants with intact function (9). Findings in our animal model support the clinical observations and the hypothesis that inflammation-related injury to the developing hippocampus can be an underlying mechanism that leads to persistent cognitive impairment in children born prematurely and have suffered sustained postnatal systemic inflammation. The hippocampus is a dynamic site of active neurogenesis that connects to all cortical regions and plays a central role in brain growth and development and in recovery after injury (10).

Repeated postnatal exposure to LPS can lead to hippocampal injury through a number of potential mechanisms outlined in Figure 3. Intraperitoneal injection of LPS can induce local peritoneal inflammation and ascites (24). Mice injected with LPS often exhibited a shiny and full abdomen suggestive of ascites. Peritoneal inflammation also leads to decreased suckling and impaired intestinal function (25). Malabsorption and malnutrition often contribute to suboptimal somatic and 
brain growth, perhaps by interfering with multiple signaling pathways (e.g., IGF (insulin-like growth factor)-1 and brainderived neurotrophic factor (BDNF)). Mice that died following LPS exposure often appeared wasted. Moreover, systemic exposure to LPS can induce a systemic inflammatory response, sepsis, and shock which can lead to impaired cerebral perfusion. Systemic inflammation can in turn induce neuroinflammation that sensitizes the brain to ischemic injury and further propagates brain damage (26). Any of these insults, either alone or in combination, can lead to subsequent brain damage or impaired recovery including but not limited to chronic inflammation and impaired synaptogenesis and neurogenesis in the developing hippocampus.

Our current experiments cannot determine the exact contribution of each possible pathway from inflammation to the brain injury described above. In addition, the present study has other limitations. First, the duration of exposure to LPS spanned a rather wide developmental window in juvenile mice. The heightened potency of the ultrasonicated LPS suspension we used in the present study allows for a targeted approach with a narrower window in future studies. Second, T2-imaging yields typically poor image detail due to the high water content of early postnatal brain tissue and the extremely small size of brain structures (27). The study focused a priori on measuring hippocampal formation area on MRI. Hence, the coordinates for the high-resolution MRI imaging of nonenhanced brain tissue used here were specifically tailored to obtain a degree of contrast on a T2-weighted image sufficient to demarcate the hippocampal formation on each slide. The coordinates of MRI imaging derived from the ex vivo nonenhanced imaging protocol described can be applied in future studies as a basis for a focused imaging approach of the developing hippocampus in vivo, and may yield sufficient contrast to allow longitudinal studies of the developing brain in live animals, which is an especially valuable tool to study genetically modified mice.

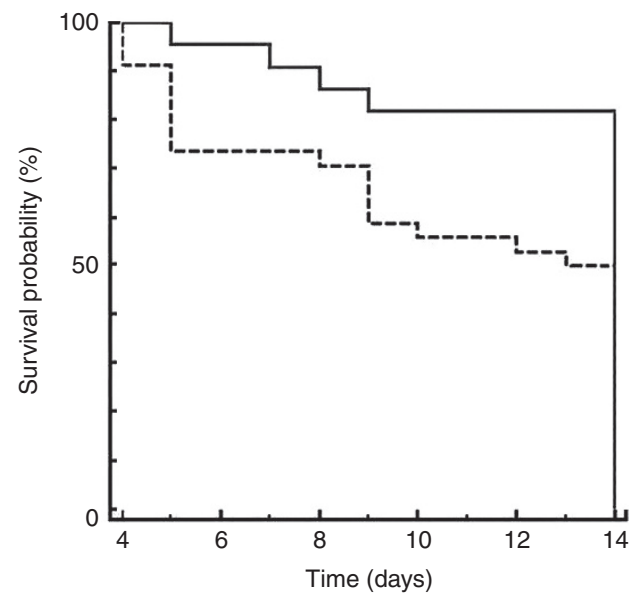

Figure 4. Kaplan-Meier estimator of survival as a function of time for mice injected daily with either saline or lipopolysaccharide (LPS) between postnatal days 3 and 13 . Approximately $50 \%$ of newborn mice survived to day 14 when injected daily with $0.3 \mathrm{mg} / \mathrm{kg}$ of LPS (dotted line) compared with $83 \%$ of mice injected with saline (solid line; $P<0.05$ ). The death rate was comparable among the litters in the LPS group.
High-resolution MRI of small animals is a valuable tool to investigate brain maturation and to delineate abnormal neuroanatomy $(27,28)$. The present study is among the first to use high-resolution small-animal MRI to show a reduction in hippocampal volume following repeated systemic exposure to LPS in the newborn period. The study also applies a model of sustained postnatal systemic inflammation in mice that mimics the clinical scenario seen in preterm infants who are at highest risk for developmental impairment (13). The experiments described allow for a quantifiable and reproducible targeted approach to investigate hippocampus-dependent impairment in rodents that mirrors working memory deficits noted in preterm infants. Moreover, the results show robust concordance between MRI, histology, and neurobehavioral testing, focusing on the developing hippocampus in mice. We believe that our findings lay the foundation for the design of specific experimental paradigms and gene modifications to further elucidate the mechanisms of inflammation-related developmental brain injury.

In conclusion, our study establishes an inflammation-based model of developmental brain injury in mice that is both quantifiable and reproducible and that mimics working memory deficits seen in extremely preterm infants. Specifically, the study produced a neurobehavioral phenotype similar to what is observed in recent follow-up cohorts of very preterm infants, i.e., significant cognitive deficits in working memory with minimal motor impairment. To our knowledge, this is the first study to show that postnatal sustained systemic inflammation can adversely affect the developing hippocampus in mice with functional impairment that lasts well into adulthood.

\section{METHODS}

\section{Animals}

Male and female C57BL6/J mice were housed in alternating 10-14-h dark-light cycles at a constant temperature $\left(20 \pm 2{ }^{\circ} \mathrm{C}\right)$ and allowed free access to standard chow and water. Inborn litters were randomly assigned to receive daily intraperitoneal injections of either phosphate-buffered saline (PBS) solution, or LPS (Sigma-Aldrich, Saint Louis, MO; cat\# L6529) dissolved in PBS from postnatal days 3 to 13 (Figure 4). The LPS was purified from E. coli 055:B5, $\gamma$-irradiated, and tested in cell culture to be free of live bacteria. LPS powder was reconstituted and ultrasonicated to ensure homogeneity in suspension. LPS was handled and injected using an aseptic technique. Mice received $0.3 \mathrm{mg} / \mathrm{kg}$ of LPS or an equivalent volume of PBS solution. The dose of LPS was derived from the literature and tested to achieve $50 \%$ survival at postnatal day 14 (14). A 50\% lethality dose was chosen to achieve appreciable morbidity in a significant number of survivors. Surviving pups were kept with their mothers until kill or weaning at $3 \mathrm{wk}$ of age. Brain and body weights, and hippocampal formation volumes and areas were measured on day 14, whereas hippocampusdependent working memory cognitive performance was examined in adulthood as detailed below. All of the procedures were approved by the Institutional Animal Care and Use Committee at Tufts University and were in accordance with the National Institutes of Health Guide for the Care and Use of Laboratory Animals.

\section{Magnetic Resonance Imaging}

Whole-brain samples from each group were suspended in $2 \%$ agarose solution and allowed to settle overnight. Each brain was then imaged ex vivo with a Bruker's PharmaScan (Burker BioSpin, Billarica, MA) 7-T magnetic resonance small-animal imaging system using a bird cage coil. The brain was imaged over a $6.5-\mathrm{h}$ period with a nonenhanced 
a

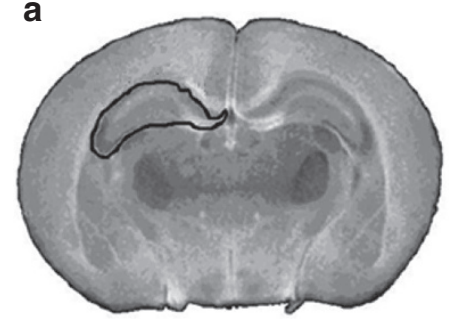

b

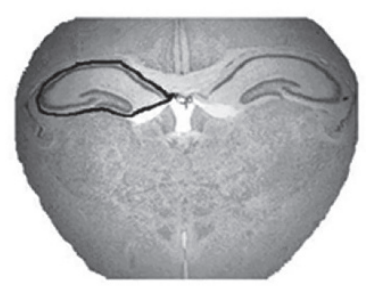

Figure 5. (a) Representative magnetic resonance (MR) image illustrating a nonenhanced coronal T2-weighted 3D RARE sequence reconstruction of a $512 \times 512 \times 512$ matrix with an in-plane resolution of $27 \mu \mathrm{m}$ and (b) a light microscopy image of a 30- $\mu \mathrm{m}$ Cresyl Violet-stained tissue section showing the hippocampal formation as the region of interest manually demarcated and its area measured using Image J software $(\mathrm{NIH})$.

coronal T2-weighted 3D RARE sequence $(\mathrm{TR}=3,000 \mathrm{~ms}$; effective $\mathrm{TE}=65 \mathrm{~ms}$; echo train length $=8$; FOV: $15.8 \times 15.8 \times 14 \mathrm{~mm}$; matrix size: $320 \times 320 \times 64$; averages $=3$ ). Reconstruction of the images to a $512 \times 512 \times 512$ matrix resulted in a slice in plane resolution of $27 \mu \mathrm{m}$. The hippocampal formation in each image was manually demarcated and its area measured using Image J software (US National Institutes of Health, Bethesda, MD; Figure 5a). The volume of the hippocampus in each brain was calculated by summation of the hippocampal formation area in all sequential images from that brain multiplied by the section thickness $(27 \mu \mathrm{m})$. Imaging and analysis were performed by personnel blind to treatment groups.

\section{Microscopy}

Brains were harvested on day 14 then fixed ex vivo in $4 \%$ paraformaldehyde and cryoprotected in a $30 \%$ sucrose solution. Whole-brain specimens were flash frozen in liquid nitrogen and 30- $\mu \mathrm{m}$-thick coronal cryostat sections were obtained. Brain sections containing the hippocampus were stained with Cresyl Violet and visualized using a Leica light microscope. Images obtained were processed using NISElements software (Nikon Instruments, Melville, NY). Images of two brain sections from comparable rostral and caudal levels were selected from each PBS- or LPS-treated animal for comparison. The hippocampal region was manually demarcated and its area was measured using Image J software $(\mathrm{NIH})$. The area of the hippocampus was calculated by adding the areas of both the right and left hippocampal regions in rostral and caudal sections from each brain (Figure $5 \mathbf{b}$ ).

\section{Behavioral Analysis}

Mice were tested between 8 and $9 \mathrm{wk}$ of age in a sequential battery of neurobehavioral tests that included Rota-Rod, spontaneous alternation on a T-maze, novel object recognition (NOR), and CFR tests. The Rota-Rod test was performed initially to screen for potential motor deficits that may confound interpretation of cognitive tests. Spontaneous alternation, NOR, and CFR tests of working memory were subsequently performed to evaluate cognitive performance.

Rota-Rod test. The Rota-Rod test assesses motor coordination and balance (29). It consists of a training session on day 1 and a testing session on day 2. On each day, animals were allowed to habituate for at least $60 \mathrm{~min}$ to the testing room, with the apparatus wiped with $70 \%$ ethanol between animals to eliminate olfactory cues. The training session consisted of three trials, $5 \mathrm{~min}$ each at a fixed speed of $16 \mathrm{rpm}$, to habituate the mice to the task. On the following day, mice were tested for three trials under accelerating speed conditions in which the rotations per minute (rpm) increased gradually from 4 to $40 \mathrm{rpm}$ over a period of $5 \mathrm{~min}$. A rest period of $30 \mathrm{~min}$ was imposed between the trials to avoid failure due to fatigue. Failure was defined as falling or as passive rotation when clinging to the rod for two full rounds. Latency to fall was recorded for each trial and the means of the three trials were compared between the groups.

Spontaneous alternation on a T-maze. This is a test of working memory that prompts the animal to explore a novel environment (15).
The mice were allowed to habituate for $60 \mathrm{~min}$ in the testing room each day before the experiments. The "T"-shaped maze consisted of alleys $12 \mathrm{~cm}$ wide and $40 \mathrm{~cm}$ long. Mice were tested in a single twotrial session. For the first trial (training), the mouse was placed at the base of the $\mathrm{T}$ and allowed to explore any of the two arms. Once the mouse fully entered an arm, it was isolated for $30 \mathrm{~s}$, after which it was returned to the starting area at the base of the T. For the second trial (test), the mouse was released and again allowed to explore any of the two arms. If the previously unexplored arm was chosen during the test trial, the choice was recorded as an "alternation". A score of $50 \%$ thus reflects absence of short-term recollection of recent environmental cues. Spontaneous alternation is sensitive to dysfunction of the hippocampus, but other brain structures are also involved, including the cerebellum, thalamus, substantia innominata, and olfactory and vestibular systems (15). However, it is rare for animals with significant hippocampal lesions to score above $60 \%$, whereas normal controls generally achieve at least $80 \%$ correct alternation and often higher (15). Alternation was recorded for each trial and the means of five trials per animal were compared between the groups.

Novel object recognition. Object recognition is considered to be driven by novelty detection in rodents (30). Mice have a tendency to interact more with a novel object than with a familiar object. NOR tests a nonmatching sample object learning task that requires intact recognition memory through the hippocampus as well as other regions of the brain (30). We conducted a one environment and two sample objects NOR testing on two consecutive days. The mice were allowed to habituate for $60 \mathrm{~min}$ in the testing room each day before the experiments. The apparatus consisted of a $36 \times 12 \times 10 \mathrm{~cm}$ rectangular white arena, two identical gray plastic cylinders, and two empty glass salt shakers. The entire apparatus was cleaned with $70 \%$ alcohol between animals to eliminate olfactory cues. On day 1 , animals were allowed to explore the empty chamber (habituation) for $10 \mathrm{~min}$. On day 2, the mice were placed for $10 \mathrm{~min}$ in the same environment with the same two objects, one in each corner (same object exposure). After a 1-h delay, the animal was placed in the same environment for $5 \mathrm{~min}$, but a novel object replaced one of the two familiar objects (novel object exposure). Location of the novel object was counterbalanced to avoid the potential confounder of side preference. Behavior was recorded using a digital camera while the experimenter remained outside the testing room. Exploration time was defined as the time each animal spent either pointing its nose toward the object within a distance of $<1 \mathrm{~cm}$ and/or touching it with its nose. NOR was scored by preferential exploration of the novel object using a discrimination factor $=$ novel object interaction time/total interaction time with both objects. The discrimination factor thus ranges from 0 to 1 , with 0.5 indicating no preference. Discrimination was recorded for each trial and a mean of six trials per animal was compared between groups.

Contextual fear conditioning. This behavioral paradigm tests the ability of adult mice to learn and recall an association between a novel environment (context) and a negative stimulus (electrical foot shock) (31). It is a form of Pavlovian associative learning that is acquired rapidly and may be long lasting (32). The brain circuitry regulating CFR is dependent on (1) the amygdala to perceive fear and (2) the hippocampus to learn and associate the context in which the fearful event took place. The apparatus consisted of a test chamber (Habitest System; Coulbourn Instruments, Whitehall, PA) with a grid floor through which scrambled electric current was delivered and a house light that was illuminated during the session. Each test chamber had a dedicated overhead camera and was enclosed within a light- and sound-attenuating cabinet. We conducted CFR testing over two consecutive days. On day 1 , the mouse was brought to the room and placed in the chamber for $5 \mathrm{~min}$. Baseline locomotion was recorded as midline chamber crossovers. After $2 \mathrm{~min}$ of acclimation, three $0.7-\mathrm{mA}$ electric shocks were delivered at 1-min intervals (acquisition). Twenty-four hours later, the mouse was placed in the same chamber for $5 \mathrm{~min}$ without shock (retrieval). Freezing during the acquisition and retrieval phases indicates fear in anticipation of the painful stimulus. Freezing episodes were recorded using FreezeFrame software and later analyzed using FreezeView software (Actimetrics, Wilmette, IL). 


\section{Statistical Analysis}

Statistical analyses were performed using Systat software (Chicago, IL) for Student's $t$-test and two-way ANOVA with Fisher's LSD post hoc analysis when applicable. Statistical significance was defined at a $P$ value of 0.05 .

\section{ACKNOWLEDGMENTS}

We thank Marelena Preda and Peter Marks at the Center for Molecular Medicine at Maine Medical Center Research Institute for their valuable technical assistance in obtaining the MR images analyzed in this study.

\section{STATEMENT OF FINANCIAL SUPPORT}

Pierre Gressens, Associate Editor-in-Chief, handled the peer review/decision-making process for this article. This study was in part supported by the Mouse Transgenic and In Vivo Imaging Core Facility supported by US National Institutes of Health grant P30GM103392 (R. Friesel, PI), Phase III COBRE in Vascular Biology.

Disclosure: The authors have no conflicts of interest to disclose.

\section{REFERENCES}

1. McCormick MC, Litt JS, Smith VC, Zupancic JA. Prematurity: an overview and public health implications. Annu Rev Public Health 2011;32:367-79.

2. Hamilton BE, Martin JA, Ventura SJ. Births: preliminary data for 2012. Natl Vital Stat Rep 2013;62:1-20.

3. Aarnoudse-Moens CS, Duivenvoorden HJ, Weisglas-Kuperus N, Van Goudoever JB, Oosterlaan J. The profile of executive function in very preterm children at 4 to 12 years. Dev Med Child Neurol 2012;54:247-53.

4. Aarnoudse-Moens CS, Weisglas-Kuperus N, van Goudoever JB, Oosterlaan J. Meta-analysis of neurobehavioral outcomes in very preterm and/or very low birth weight children. Pediatrics 2009;124:717-28.

5. Delobel-Ayoub M, Arnaud C, White-Koning M, et al.; EPIPAGE Study Group. Behavioral problems and cognitive performance at 5 years of age after very preterm birth: the EPIPAGE Study. Pediatrics 2009;123:1485-92

6. Griffiths ST, Gundersen H, Neto E, et al. fMRI: blood oxygen level-dependent activation during a working memory-selective attention task in children born extremely preterm. Pediatr Res 2013;74:196-205.

7. Herschkowitz N, Kagan J, Zilles K. Neurobiological bases of behavioral development in the first year. Neuropediatrics 1997;28:296-306.

8. Müller NG, Knight RT. The functional neuroanatomy of working memory: contributions of human brain lesion studies. Neuroscience 2006;139:51-8.

9. Beauchamp MH, Thompson DK, Howard K, et al. Preterm infant hippocampal volumes correlate with later working memory deficits. Brain 2008;131(Pt 11):2986-94.

10. Miles DK, Kernie SG. Activation of neural stem and progenitor cells after brain injury. Prog Brain Res 2006;157:187-97.

11. Malaeb S, Dammann O. Fetal inflammatory response and brain injury in the preterm newborn. J Child Neurol 2009;24:1119-26.

12. Dammann O. Persistent neuro-inflammation in cerebral palsy: a therapeutic window of opportunity? Acta Paediatr 2007;96:6-7.

13. Dammann O, Leviton A. Intermittent or sustained systemic inflammation and the preterm brain. Pediatr Res 2014;75:376-80.

14. Wang X, Hellgren G, Löfqvist C, et al. White matter damage after chronic subclinical inflammation in newborn mice. J Child Neurol 2009;24:1171-8.

15. Deacon RM, Rawlins JN. T-maze alternation in the rodent. Nat Protoc 2006;1:7-12.
16. Du X, Fleiss B, Li H, et al. Systemic stimulation of TLR2 impairs neonatal mouse brain development. PLoS One 2011;6:e19583.

17. Favrais G, van de Looij Y, Fleiss B, et al. Systemic inflammation disrupts the developmental program of white matter. Ann Neurol 2011;70:550-65.

18. Harré EM, Galic MA, Mouihate A, Noorbakhsh F, Pittman QJ. Neonatal inflammation produces selective behavioural deficits and alters N-methyl$\mathrm{D}$-aspartate receptor subunit mRNA in the adult rat brain. Eur J Neurosci 2008;27:644-53.

19. Wang KC, Fan LW, Kaizaki A, Pang Y, Cai Z, Tien LT. Neonatal lipopolysaccharide exposure induces long-lasting learning impairment, less anxiety-like response and hippocampal injury in adult rats. Neuroscience 2013;234:146-57.

20. Lowe GC, Luheshi GN, Williams S. Maternal infection and fever during late gestation are associated with altered synaptic transmission in the hippocampus of juvenile offspring rats. Am J Physiol Regul Integr Comp Physiol 2008;295:R1563-71.

21. Smith PL, Hagberg H, Naylor AS, Mallard C. Neonatal peripheral immune challenge activates microglia and inhibits neurogenesis in the developing murine hippocampus. Dev Neurosci 2014;36:119-31.

22. Leviton A, Kuban KC, Allred EN, Fichorova RN, O'Shea TM, Paneth N; ELGAN Study Investigators. Early postnatal blood concentrations of inflammation-related proteins and microcephaly two years later in infants born before the $28^{\text {th }}$ post-menstrual week. Early Hum Dev 2011;87: 325-30.

23. O'Shea TM, Allred EN, Kuban KC, et al.; Extremely Low Gestational Age Newborn (ELGAN) Study Investigators. Elevated concentrations of inflammation-related proteins in postnatal blood predict severe developmental delay at 2 years of age in extremely preterm infants. J Pediatr 2012;160: 395-401.e4.

24. Kopydlowski KM, Salkowski CA, Cody MJ, et al. Regulation of macrophage chemokine expression by lipopolysaccharide in vitro and in vivo. J Immunol 1999;163:1537-44.

25. Deitch EA, Xu D, Kaise VL. Role of the gut in the development of injuryand shock induced SIRS and MODS: the gut-lymph hypothesis, a review. Front Biosci 2006;11:520-8.

26. Eklind S, Mallard C, Leverin AL, et al. Bacterial endotoxin sensitizes the immature brain to hypoxic-ischaemic injury. Eur J Neurosci 2001;13:11016.

27. Baloch S, Verma R, Huang H, et al. Quantification of brain maturation and growth patterns in C57BL/6 J mice via computational neuroanatomy of diffusion tensor images. Cereb Cortex 2009;19:675-87.

28. Cengiz P, Uluc K, Kendigelen P, et al. Chronic neurological deficits in mice after perinatal hypoxia and ischemia correlate with hemispheric tissue loss and white matter injury detected by MRI. Dev Neurosci 2011;33: $270-9$.

29. Jones BJ, Roberts DJ. The quantitative measurement of motor inco-ordination in naive mice using an accelerating rotarod. J Pharm Pharmacol 1968;20:302-4.

30. Bevins RA, Besheer J. Object recognition in rats and mice: a one-trial nonmatching-to-sample learning task to study 'recognition memory'. Nat Protoc 2006;1:1306-11.

31. Wehner JM, Radcliffe RA. Cued and contextual fear conditioning in mice. Curr Protoc Neurosci 2004; Chapter 8:Unit 8.5C.

32. Maren S. Neurobiology of Pavlovian fear conditioning. Annu Rev Neurosci 2001;24:897-931. 